\title{
Superior vena cava syndrome secondary to indwelling peripherally inserted perm-catheter in 33-year-old female
}

\author{
Bankim Patel ${ }^{1}$, Aravindan Jeyarjasingam² ${ }^{2}$ Kunal Patel ${ }^{2}$, Rupen Patel $^{3}$, Gaurav Agarwal ${ }^{2}$ \\ 1. Ross University, School of Medicine, USA. 2. Mount Sinai Hospital Internal Medicine Residency Program, Chicago, IL, \\ USA. 3. East Carolina University/Brody School of Medicine, USA
}

Correspondence: Bankim Patel. Address: Ross University School of Medicine, USA. Email: bankim.patel19@gmail.com

Received: August 10, 2015

DOI : $10.5430 /$ crim.v2n4p51

Accepted: September 27, 2015

Online Published: October 12, 2015

\begin{abstract}
We report a case of acute onset superior vena cava (SVC) syndrome secondary to long-term indwelling catheter, utilized for dialysis in a patient during a previous hospital stay, and a review of literature. The patient is a 33-year-old female with a past medical history of Type 1 diabetes, hypertension, end stage renal disease, cerebral vascular accident (CVA), and peripheral artery disease presenting with acute onset of facial, neck, and upper extremity swelling with decreased oxygen saturation. Symptoms of SVC syndrome include swelling of the face, neck, and upper extremity. In addition, there is prominent facial edema and flushing, with jugular venous distension that is exacerbated when the arms are placed over the head. Diagnosis includes a thorough clinical exam with imaging modalities for confirmative diagnosis. Options include noninvasive imaging such as ultrasound, which is preferred for peripheral venous obstruction, while magnetic resonance angiography (MRA) and computed tomography angiography (CTA) can confirm central obstruction. Treatment due to stenosis by catheter involves stenting with balloon angioplasty which can provide immediate relief of symptoms. High degree of suspicion for superior vena cava syndrome should arise when a patient presents with common symptoms and long-term central lines. This case demonstrates the long-term effects of indwelling catheters on patients with pre-existing vascular and renal disease.
\end{abstract}

\section{Keywords}

Superior vena cava syndrome, Permacath, Hemodialysis, Stenosis, Catheter

\section{Case presentation}

We report a case of acute onset superior vena cava (SVC) syndrome secondary to a long-term indwelling catheter utilized for dialysis in a patient during a previous hospital stay. The patient is a 33-year-old female with a past medical history of Type 1 diabetes, hypertension, end stage renal disease (ESRD) on dialysis three times a week, right posterior cerebral artery CVA in 2013, gastroparesis and peripheral artery disease presented with altered mental status, acute onset of facial, neck, and upper extremity swelling with intermitted episodes of decreased oxygen saturation for approximately a one week duration. In addition, she arrived from the rehabilitation hospital with a right-sided subclavian Perm-a-Cath that was present for approximately one month prior to admission for dialysis access. The time frame for perm-a-cath placement was unknown; however, she was previously diagnosed with ESRD for an extended amount of time without any consideration 
for alternate means for long-term dialysis access prior to hospital admission. Prior to admission, she had a CT scan of the brain, which demonstrated resolving subarachnoid hemorrhage which justified the absence of anticoagulation during hospital stay. She was not started on heparin nor was she on anticoagulation prior to admission. The patient's coagulation panel was obtained with an IN-0.8, PT-9.4, and PTT-34.1. Her presentation was positive for confusion and lower extremity swelling and negative for orthopnea, cough, stridor, or palpitations.

She has a negative social history and reported an allergic reaction to aspirin and nonsteroidal anti-inflammatory drugs which caused nausea and vomiting. In addition, she was on lisinopril 2.5 daily at the time of admission. On physical examination, she was lethargic but arousable. The patient's cranial exam demonstrated right facial, eye, and preauricular swelling. The patient's pulses were present bilaterally within the upper and lower extremities and lymphatic exam was unremarkable.

Following clinical examination, we suspected a reaction to lisinopril and discontinued the use of ace inhibitors without any improvement in symptoms. We performed a CT scan of the head and neck, which demonstrated diffused facial neck edema and increased retropharyngeal space with diffuse retropharyngeal edema. During hospital stay, she required a peripherally inserted central catheter (PICC) for dialysis since the Perm-a-cath was suspected as a cause for edema.

She continued to have worsening edema with extension down to the chest; however, there were no signs of stridor or compromise in airway. A repeat CT scan of head and neck demonstrated a $2 \mathrm{~mm}$ narrowing at the level of the false cords of the trachea (see Figure 1). Consequently, she underwent a CT angiogram that demonstrated possible occlusion of the right subclavian and jugular veins with the right PICC line and Perm-a-cath present (see Figure 2). The central lines were both present in the SVC prior to beginning of the right atrium. Therefore, the Perm-a-Cath was removed and she underwent SVC and subclavian angiogram with angioplasty. The bilateral arm venogram confirmed focal stenosis of the SVC with filling defects of the right innominate vein. She subsequently underwent angioplasty with a $12 \mathrm{~mm}$ diameter balloon catheter with resolution of her symptoms and reestablishment of flow within the superior vena cava. A stent was not placed since there was significant improvement of venous return from the head, neck, and upper limbs. We cannot comment on reasoning behind placing the catheter in the SVC.
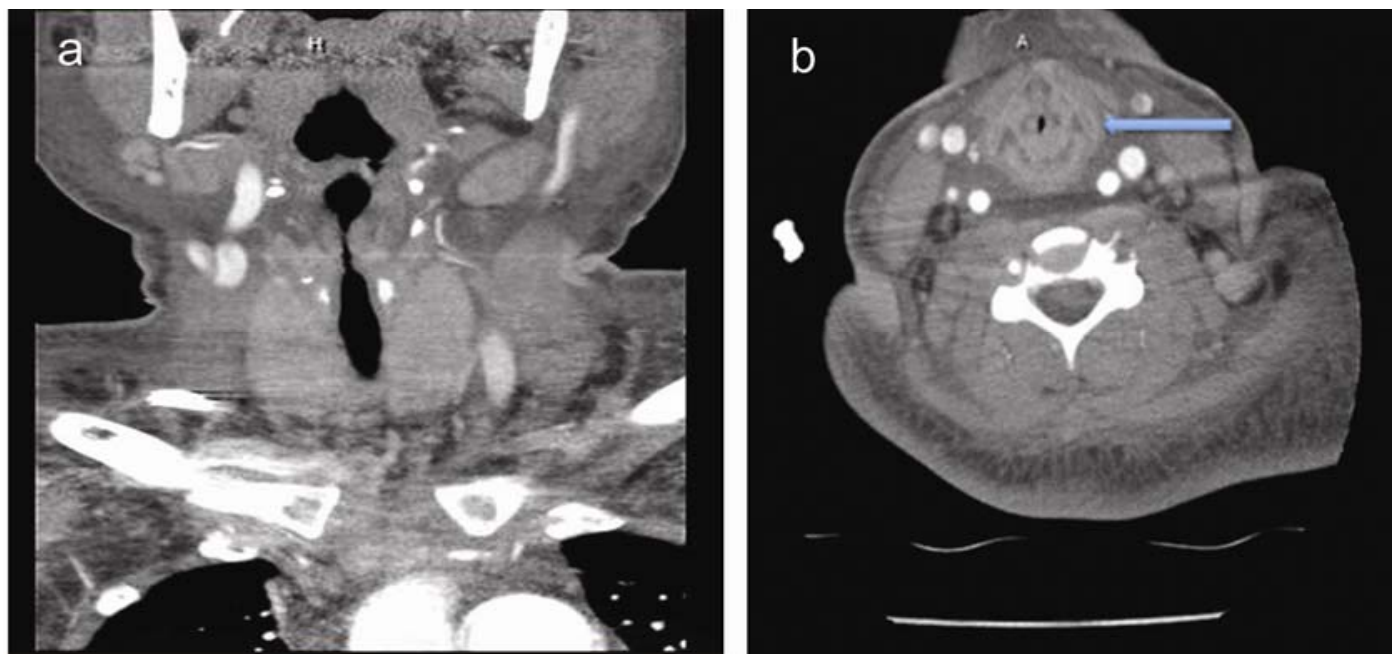

Figure 1. CT Imagining studies. Arrows demonstrating CT Neck views showing diffuse edema with tracheal narrowing at the level of the false vocal cords measuring approximately $2 \mathrm{~mm}$

\section{Discussion}

SVC syndrome is historically associated with malignancy tumors or primary bronchial carcinomas in the upper mediastinum in the majority of cases. The minority of cases have non-malignant causes including tuberculosis, 
histoplasmosis, actinomycosis, stenosis, and thrombosis ${ }^{[1,2]}$. Recently, the usage of long-term indwelling catheters and pacemakers has led to an increasing occurrence of superior vena cava syndrome due to thrombogenic and stenotic causes. Our patient's time frame for perm-a-cath placement was approximately one month however. Upon literature review, the nonmalignant causes may represent up to $40 \%$ of cases of SVC syndrome - to date no causes were determined due to indwelling perm-a-catheters ${ }^{[2]}$.

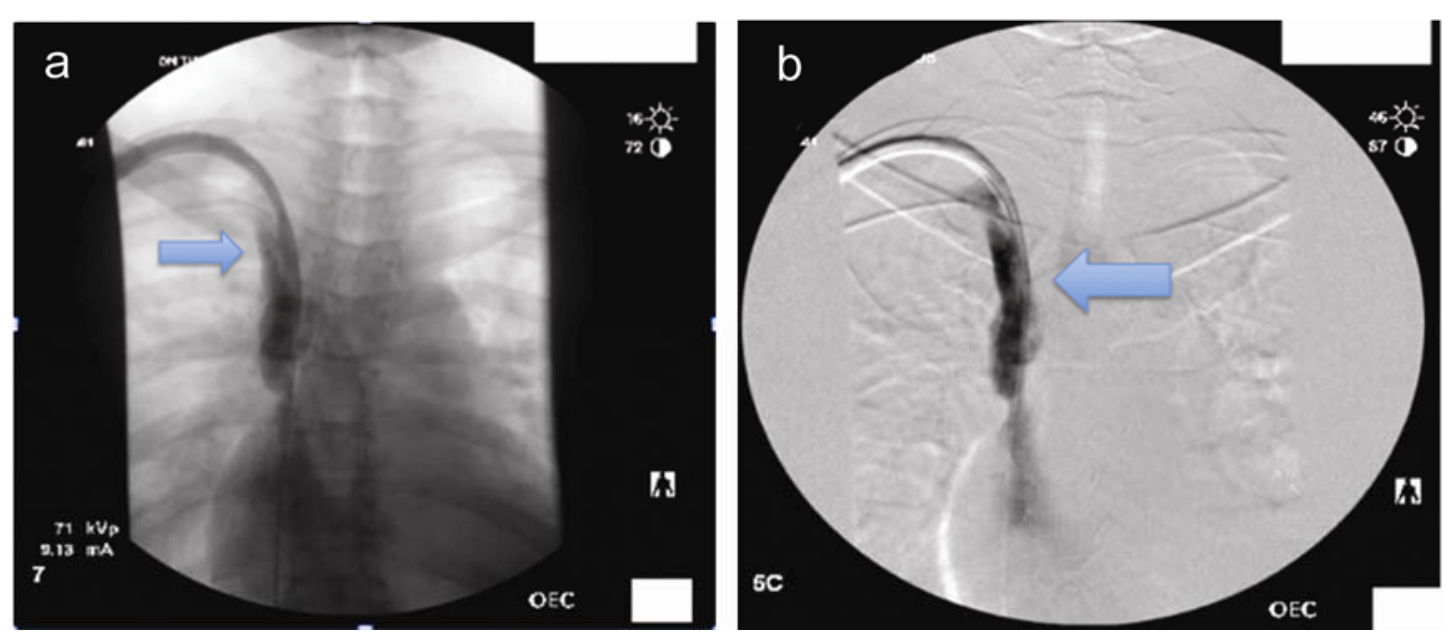

Figure 2. Venography. Arrows highlighting venography demonstrating significant SVC narrowing with poor anterograde flow

Central venous catheters and pacemakers are the most common causes due to indwelling device. In addition, subclavian vein catheterization is associated with stenosis, thrombosis, infection, hemo-pneumothorax, and bleeding ${ }^{[3,4]}$. Our patient was especially at risk for bleeding due to her end-stage renal disease leading platelet dysfunction. Virchows triad provides the potential internal reasons towards the cause of acute superior vena cava syndrome due to thrombosis ${ }^{[5]}$. However, we must consider other internal causes with the patient having indwelling catheters. Catheters can cause trauma to the endothelium or high rate and pressure of blood flow during hemodialysis are proposed mechanisms for stenosis ${ }^{[6]}$. Our patient's speculated cause was due to vein fibrosis and collapse due to long term use of the Perm-a-Cath. Causes by external compression in the differential diagnosis with patients having negative chest imaging or absence of catheters must be included in the differential. In addition, congestive heart failure and angioedema can present with similar symptoms to SVC $^{[7]}$.

These patients usually present with acute manifestations due to the lack of venous collateral development and the rapid development of either vein collapse or thrombosis ${ }^{[1]}$. Whereas the gradual formation of obstruction secondary to malignancy allows development of vascular formation which prevents clinical symptoms. Upon literature review, symptoms of SVC syndrome include swelling of the face, neck, and upper extremity. In addition, there is prominent facial edema and flushing, with jugular venous distension that is exacerbated when the arms are placed over the head. In addition, more severe findings can include dysphagia, stridor, periorbital edema, chest pain, headache, changes in mental status, and syncope. The SVC is susceptible to thrombengenic diathesis due to the thin wall with low venous pressures while being anatomically constricted making it easily compressible by nearby structures. The promptness of symptoms and signs from SVC obstruction is collar of the rate of obstruction leading to completed blockage of the SVC with consideration of the recruitment of venous collaterals.

Our patient developed symptoms within two days, which favors a diagnosis of thrombosis with combination of fibrosis and venous collapse of the right upper extremity drainage. A right upper extremity ultrasound of our patient demonstrated a thrombus of the right subclavian and axillary veins.

Diagnosis includes a thorough clinical exam with imaging modalities for confirmative diagnosis. Options include noninvasive imaging such as ultrasound, which is preferred for peripheral venous obstruction; while magnetic resonance 
angiography (MRA) and computed tomography angiography (CTA) can confirm central obstruction and are noninvasive. Contrast venography is another modality for diagnosis; however, its invasive nature and the use of dyes limits usage. Treatment due to stenosis by catheter involves stenting with balloon angioplasty which can provide immediate relief of symptoms while patients with obstruction secondary to thrombus are treated through anticoagulation with low molecular weight heparin or thrombolytics.

\section{Conclusion}

High degree of suspicion for superior vena cava syndrome should arise when a patient presents with common symptoms and long-term central lines. This case demonstrates the long-term effects of indwelling catheters on patients with pre-existing vascular and renal disease. A thorough patient history allows the clinician to anticipate other diagnosis and institute the appropriate medical management.

\section{References}

[1] Shaheen K, Alraies MC. Superior vena cava syndrome. Cleveland Clinic Journal of Medicine. 2012; 79(6): 410-412. PMid:22660872 http://dx.doi.org/10.3949/ccjm.79a.11106

[2] Wilson LD, Detterbeck FC, Yahalom J. Superior vena cava syndrome with malignant causes. The New England Journal of Medicine. 2007; 356(18): 1862-1869. PMid:17476012 http://dx.doi.org/10.1056/NEJMcp067190

[3] Bander SJ, Schwab SJ. Central venous angioaccess for hemodialysis and its complications. Semin Nephrol. 1985 ; 5: $121-8$.

[4] Vanholder R, Lameire N, Verbanck J, et al. Complications of subclavian catheter hemodialysis: a 5 year prospective study in 257 consecutive patients. Int J Artif Organs. 1982; 5: 297-303. PMid:7174135

[5] Warren P, Burke C. Endovascularmanagement of chronic upper extremity deep vein thrombosis and superior vena cava syndrome. Seminars in Interventional Radiology. 2011; 28(1): 32-38. PMid:22379274 http://dx.doi.org/10.1055/s-0031-1273938

[6] Khor T, Anderson J, McRae P. Central venous thrombophlebitis diagnosed by computerized tomography scanning. Aust NZ J Surg. 1992; 62: 820-2. PMid:1445066 http://dx.doi.org/10.1111/j.1445-2197.1992.tb06928.x

[7] Pennell N, Hodgson JM, Bahler R, et al. Superior Vena Cava Syndrome. Elsevier. 2013. 